\title{
The accessible promoter-mediated supplementary effect of ACE2 provides new insight into the tissue tropism of SARS-CoV-2
}

\section{Qi Jiang}

Tongfang Cloud (Beijing) Technology Co., Ltd

\section{Guifang Du}

Beijing Institute of Radiation Medicine

Junting Wang

The First Affiliated Hospital of Harbin Medical University

\section{XiaoHan Tang}

The First Affiliated Hospital of Harbin Medical University

\section{Xuejun wang}

Beijing Institute of Radiation Medicine

\section{Ding Yang}

Beijing Institute of Radiation Medicine

Fei Li

Computer Network Information Center, Chinese Academy of Sciences

\section{Yu Sun}

Beijing Institute of Radiation Medicine

\section{Huan Tao}

Beijing Institute of Radiation Medicine

\section{Meisong Lu}

The First Affiliated Hospital of Harbin Medical University

Hao Li

Beijing Institute of Radiation Medicine

Hebing Chen

Beijing Institute of Radiation Medicine

Xiaochen Bo ( $\square$ boxc@bmi.ac.cn )

Beijing Institute of Radiation Medicine

\section{Research Article}

Keywords: Angiotensin-converting enzyme 2 (ACE2), SARS-CoV-2, COVID-19 
Posted Date: December 11th, 2020

DOI: https://doi.org/10.21203/rs.3.rs-117172/v1

License: (c) (i) This work is licensed under a Creative Commons Attribution 4.0 International License. Read Full License 
1 The accessible promoter-mediated supplementary effect of

2 ACE2 provides new insight into the tissue tropism of SARS-

\section{$\mathrm{CoV}-2$}

4 Qi Jiang ${ }^{2, \dagger}$, Guifang Du ${ }^{1, \dagger}$, Junting Wang ${ }^{3, \dagger}$, XiaoHan Tang ${ }^{3}$, Xuejun Wang ${ }^{1}$, Yang Ding ${ }^{1}$, Fei Li ${ }^{4}$, Yu

5 Sun ${ }^{1}$, Huan Tao ${ }^{1}$, Meisong $\mathrm{Lu}^{3}$, Hao Li ${ }^{1, *}$, Hebing Chen ${ }^{1, *}$ and Xiaochen Bo ${ }^{1, *}$

61 Beijing Institute of Radiation Medicine, Beijing 100850, China.

72 Tongfang Cloud (Beijing) Technology Co., Ltd, Beijing, China.

83 The First Affiliated Hospital of Harbin Medical University, Harbin 150001, China.

94 Computer Network Information Center, Chinese Academy of Sciences, Beijing 100190, China.

*Corresponding authors: Tel: +8601066932251; Email: lihao thu@163.com(H.L.);chb-1012@163.com

11 (H.C.); $\underline{\text { boxc@bmi.ac.cn (X.B.) }}$

$12 \dagger$ These authors contributed equally to this work.

\section{Abstract}

Background: Angiotensin-converting enzyme 2 (ACE2) has been confirmed to be a receptor for the newly discovered severe acute respiratory syndrome coronavirus 2 (SARS-CoV-2). However, cell surface ACE2 expression is reported to be inconsistent with clinical tissue tropism of SARS-CoV-2, which complicates understanding of the pathogenesis of 2019 novel coronavirus disease (COVID-19). The consumption of

19 ACE2 by internalization and shedding processes may explain this discordance.

Results : To understand the discordance between ACE2 expression and the tissue tropism of SARS-CoV-2, we examined the chromatin accessibility of ACE2 promoter 
in hundreds of tissues and cell lines using public DNase-seq and assay for transposase-

23 accessible chromatin with high throughput sequencing (ATAC-seq) data. We find that

24 ACE2 promoter is only accessible in three tissues including lung, large intestine and

25 placenta. Also, we examined tumors tissues and ACE2 promoter is observed accessible

26 in five tumors with reported SARS-CoV-2 susceptibility. We confirmed the

27 susceptibility by performing SARS-CoV-2 pseudovirus infection in several cell lines.

28 Conclusions: We propose that open chromatin at the promoter mediates the ACE2

29 supplementary effect and ensures the entry of SARS-CoV-2. This hypothesis provides

30 a new view and potential clues for further investigation of COVID-19 pathogenesis.

\section{Introduction}

32 The emerging novel betacoronavirus, known as severe acute respiratory syndrome

33 coronavirus 2 (SARS-CoV-2), has caused major outbreaks in many parts of the world

34 and threatens public health [1]. The World Health Organization has declared that 2019

35 novel coronavirus disease (COVID-19), caused by SARS-CoV-2, has created a public

36 health emergency of international concern. Similarly to SARS-CoV [2, 3], SARS-CoV-

372 employs angiotensin-converting enzyme 2 (ACE2) as a receptor for cellular entry [4].

38 However, previous studies have documented generally low ACE2 expression in

39 multiple tissues, including lung and airway epithelium [5-7]. We also confirmed this

40 observation using genotype-tissue expression [8] RNA sequencing (RNA-seq) data (Fig.

41 S1 and Table S1). The discordance between ACE2 expression and SARS-CoV-2

42 tropism complicates the understanding of the infection, spread, and clearance of this 
virus. One possible explanation is that ACE2 expression is under-detected due to technical dropout effects, especially in single-cell RNA sequencing (scRNA-seq) [5].

The state of chromatin dictates fundamental cellular processes, including gene expression [9]. Accessible chromatin has long been known to mark regulatory sequences and to interact with transcription factors to execute transcriptional programs instructing cell fate determination and development $[10,11]$. The activation of genes generally correlates positively with increased promoter accessibility [12]. Thus, the examination of chromatin accessibility will help to reveal the transcriptional activity and cell state. Here, by investigating the chromatin accessibility of the ACE2 gene in multiple datasets from different tissues and cell lines, we show that the ACE2 promoter is accessible in limited tissues and cells, and that this tissue-specific pattern is quite accordant with clinical findings. We propose that open chromatin at the promoter mediates the ACE2 supplementary effect and further induces SARS-CoV-2 tropism.

\section{Methods}

\section{SARS-CoV-2 pseudovirus infection assays}

Cells were seeded at a density of $1 \times 10^{4} /$ well in 96 -well plates $16 \mathrm{~h}$ before infection. Cells in each well were then infected with SARS-CoV-2 pseudovirus expressing luciferase at a multiplicity of infection of 10 . The culture medium was changed $12 \mathrm{~h}$ post-infection, and luciferase activity was detected at $72 \mathrm{~h}$ post-infection using the Steady-Lumi ${ }^{\text {TM }}$ II Firefly Luciferase Assay Kit (Beyotime). Cell was purchased 


\section{Results}

The ACE2 promoter is accessible in limited human tissues, including lung tissue

As previously reported, ATAC-seq and DNase-seq approach as been recognized as the best measures of chromatin accessibility [13]. To investigate the chromatin accessibility of ACE2, we developed a pipeline by simply mapping ATAC-seq peaks and DNase I hypersensitivity sites (DHSs) to bin-based genomic regions. Briefly, genomic region chrX: 15576000-15622500 (Gencode V19), covering the ACE2 gene locus, was split by $500 \mathrm{bp} / \mathrm{bin}$, and accessible bins were defined as those overlapping with ATAC-seq peaks and DHSs by at least one base pair. We first examined ACE2 accessibility in 33 human tissue types (Table S2). A non-coding region (chrX: 1558300-1558400) was accessible in almost all tissues except those from the brain and thymus, indicating the importance of chromatin regulation by this region. In contrast, the promoter region of ACE2 (chrX: 15619500-15620000) was accessible only in lung, large intestine, and placenta tissue. These observations are consistent with the tissue tropism observed in previous studies. First, as a respiratory virus, SARS-CoV-2 targets primarily the lung. Second, unexpected diarrhea was reported in patients with COVID-19 [14], and most

81 affected patients exhibited positivity in their stool, which persisted in some patients even after the viral RNA load had decreased to an undetectable level in the respiratory tract [15]. Third, ACE2 expression is noticeable in certain placental/decidual cell types without transmembrane serine protease 2 (TMPRSS2) [5]. In addition, there are clinical 
0

reports suggesting the occurrence of vertical SARS-CoV-2 transmission, e.g. a neonate born to a mother with COVID-19 had elevated antibody levels and abnormal cytokine test results 2 hours after birth [16], an infant whose mother had been infected with SARS-CoV-2 showed positivity at $36 \mathrm{~h}$ after birth [17].

Appropriate activities of gene promoters are essential for coordinated transcription within a cell [13]. Chromatin accessibility at the promoter represents potency in terms of the ability to execute transcriptional programs. Thus, this specificity of chromatin accessibility at the ACE2 promoter site provides important hints about the tissue tropism of SARS-CoV-2 infection.

\section{The ACE2 promoter is specifically accessible in SARS-CoV-2-susceptible tumors}

In contrast to normal tissues, cancer tissues are highly heterogeneous, with each tumor type exhibiting distinct clinical features and associated with different patient outcomes and therapeutic responses [11]. The examination of chromatin accessibility enables the identification of cancer-specific DNA regulatory elements and the classification of tumor subtypes [11]. Due to the systemic immunosuppressive state caused by malignancy and anticancer treatments, patients with cancer are more susceptible to COVID-19 infection [18-20]. Thus, chromatin accessibility at the ACE2 promoter site in cancer cells needs to be investigated. We investigated ATAC-seq data for 23 types of tumor tissue from The Cancer Genome Atlas project [21]. The promoter of ACE2 was 
accessible in five (22\%) tumor tissue samples (lung adenocarcinoma, colon

107 adenocarcinoma, adrenocortical carcinoma, breast invasive carcinoma, and thyroid

108 carcinoma; Fig. 2A and Table S3); this proportion was much higher than that in normal

109 tissues (5/33 [15\%]; Fig. 1). The accessibility of the ACE2 promoter in these five tumor

110 types is consistent with a recent clinical report, which stated that 18 of 1590 individuals

111 with COVID-19 had histories of cancer, including 5 with lung cancer, 3 with breast

112 cancer, 3 with colorectal carcinoma, 1 with adrenal neoplasm, and 1 with papillary

113 thyroid microcarcinoma histories [19]. We also examined DHS data for 63 cancer cell

114 lines from the Encyclopedia of DNA Elements project [22] (Table S4). Similarly, the

115 percentage of cancer cell lines (10/63 [16\%]; Fig. 2B) with ACE2 promoter

116 accessibility was much higher than that in normal cell lines (11/126 [9\%]; Fig. 2C and

117 Table S5). Furthermore, we conducted a SARS-CoV-2 pseudovirus infection

118 experiment to examine susceptibility to this virus. Four cancer cell lines (HepG2 and

119 Caco-2 with DNase-seq peaks in the ACE2 promoter, K562 and Jurkat without) and the

120 normal cell line BHK21 (background) were infected with HIV-based pseudovirus

121 bearing the SARS-CoV-2 Spike protein. Luciferase activity in these cells was detected

122 at $72 \mathrm{~h}$ post infection. As expected, we observed that the HepG2 and Caco-2 cell lines

123 were susceptible to the SARS-CoV-2 pseudovirus, and, K562 and Jurkat were

124 unsusceptible (Fig. 2D). 
127 ACE2 has been reported to be the surface cellular receptor for SARS-CoV and SARS-

$128 \mathrm{CoV}-2$ cell entry $[23,24]$. However, as endocytosis is essential for the establishment of

129 virus entry, ACE2 is internalized together with SARS-CoV upon infection [2, 25-27].

130 In addition, ACE2 undergoes ectodomain shedding, making it subject to

131 juxtamembrane cleavage events [28, 29]. Thus, ACE2 expression on cell surfaces is

132 dynamic, with new ACE2 supplementing diminished ACE2 cell surface expression.

133 Here, we propose an accessible promoter-based model to show the potential

134 supplementary mechanism of ACE2 cell surface expression, using the lung as an

135 example (Fig. 3). Accessible chromatin at the ACE2 promoter site provides templates

136 for transcription factor binding, and transcription factors then recruit cofactors and Pol

137 II for transcription initiation. Thus, accessibility of the ACE2 promoter is required for

138 continuous transcription to compensate cell surface ACE2, which is consumed by

139 internalization and shedding processes.

140

141 Discussion

142 A fundamental phenomenon in SARS-CoV-2 infection is the discordance between

143 virus tropism and ACE2 receptor expression, as measured by RNA-seq or scRNA-

144 seq. Here, we report on a systematic survey of accessible chromatin of ACE2 in

145 hundreds of tissues and cell lines. Our results reveal that the promoter of ACE2 has

146 limited accessibility, restricted to SARS-CoV-2-susceptible tissues and cell lines. Due 
147 to the contribution of accessible chromatin to the establishment of transcriptional

148 programs, open chromatin at the ACE2 promoter site ensures the compensation of cell

149 surface ACE2. This result provides new insight into the tissue tropism of SARS-CoV-

150 2. Further investigations with additional experiments are warranted to fully confirm

151 this hypothesis.

152 Supplemental information

153 Supplemental Information can be found online at the website.

154 Acknowedgments

155 We thank the ENCODE Consortium, TCGA project, and the GTEx Portal for providing

156 high-quality data. This work was supported by the National Natural Science Foundation

157 of China (http://www.nsfc.gov.cn; nos. 31801112, 31900488, and 61873276 to HC, HL,

158 and XB, respectively), the Beijing Nova Program of Science and Technology

159 (https://mis.kw.beijing.gov.cn; no. Z191100001119064 to HC), and the Beijing Natural

160 Science Foundation (http://kw.beijing.gov.cn/; no. 5204040 to HL).

161 Author contributions

162 X. B., H. C. and H. L. conceived the project. Q.J., G.D., J.W., X.T., Y.D., F.L., Y.S., H.T.

163 and M.L. conducted the investigations and data mining. X.W. conducted the

164 pseudovirus infection experiment. Q.J., G.D. and J.W. wrote the paper.

165 Funding: 
167 (http://www.nsfc.gov.cn; no: 61873276 to X.B.), the National Natural Science

168 Foundation of China(http://www.nsfc.gov.cn; no: 31801112 to H.C.), the Beijing

169 Nova Program of Science and Technology (https://mis.kw.beijing.gov.cn; no.

170 Z191100001119064 to HC); the National Natural Science Foundation of China

171 (http://www.nsfc.gov.cn; no. 31900488 to H.L) and Beijing Natural Science

172 Foundation (http://kw.beijing.gov.cn/; no. 5204040 to H.L.)

173 Ethics approval and consent to participate

174 Not applicable

175 Consent for publication

176 Not applicable

177 Availability of data and materials

178 Not applicable

179 Competing interests

180 The authors declare that they have no competing interests

181 References

182 1. Lee Elizabeth C. WNI, Grabowski M Kate., Gurley Emily S., Lessler 183 Justin.(2020). The engines of SARS-CoV-2 spread. : The engines of SARS- 
2. Li W, Moore MJ, Vasilieva N, Sui J, Wong SK, Berne MA, Somasundaran M, Sullivan JL, Luzuriaga K, Greenough TC et al: Angiotensin-converting enzyme 2 is a functional receptor for the SARS coronavirus. Nature 2003.

3. Matsuyama S, Nagata N, Shirato K, Kawase M, Takeda M, Taguchi F: Efficient Activation of the Severe Acute Respiratory Syndrome Coronavirus Spike Protein by the Transmembrane Protease TMPRSS2. Journal of Virology 2010, 84(24):12658-12664.

192

4. Clausen TM, Sandoval DR, Spliid CB, Pihl J, Perrett HR, Painter CD, Narayanan A, Majowicz SA, Kwong EM, McVicar RN et al: SARS-CoV-2 Infection Depends on Cellular Heparan Sulfate and ACE2. Cell 2020,

5. Sungnak W, Huang N, Bécavin C, Berg M, Queen R, Litvinukova M, Talavera-

6. Zou X, Chen K, Zou J, Han P, Hao J, Han Z: Single-cell RNA-seq data analysis on the receptor ACE2 expression reveals the potential risk of different human organs vulnerable to 2019-nCoV infection. Frontiers of medicine 2020.

204 7. Qi F, Qian S, Zhang S, Zhang Z: Single cell RNA sequencing of 13 human 
tissues identify cell types and receptors of human coronaviruses. Biochemical and biophysical research communications 2020, 526(1):135-140.

207 8. Mele M, Ferreira PG, Reverter F, DeLuca DS, Monlong J, Sammeth M, Young TR, Goldmann JM, Pervouchine DD, Sullivan TJ et al: Human genomics. The human transcriptome across tissues and individuals. Science (New York, NY) 2015, 348(6235):660-665.

211 9. Kouzarides T: Chromatin Modifications and Their Function. Cell 2007, 128(4):0-705.

213 10. Rivera CM, Ren B: Mapping Human Epigenomes. Cell 2013, 155(1):39-55.

214 11. Corces MR, Granja JM, Shams S, Louie BH, Seoane JA, Zhou W, Silva TC, Groeneveld C, Wong CK, Cho SW et al: The chromatin accessibility landscape of primary human cancers. Science (New York, NY) 2018,

218 12. Fenouil R, Cauchy P, Koch F, Descostes N, Cabeza JZ, Innocenti C, Ferrier P, Genome Research 2012, 22(12):2399-2408.

222 13. Andersson R, Sandelin A: Determinants of enhancer and promoter activities of regulatory elements. Nature reviews Genetics 2020, 21(2):71-87.

224 14. Guan WJ, Ni ZY, Hu Y, Liang WH, Ou CQ, He JX, Liu L, Shan H, Lei CL, Hui DSC et al: Clinical Characteristics of Coronavirus Disease 2019 in China. 
227 15. Xiao F, Tang M, Zheng X, Li C, He J, Hong Z, Huang S, Zhang Z, Lin X, Fang $\mathrm{Z}$ et al: Evidence for gastrointestinal infection of SARS-CoV-2. medRxiv 2020.

230 16. Dong L, Tian J, He S, Zhu C, Wang J, Liu C, Yang J: Possible Vertical Transmission of SARS-CoV-2 From an Infected Mother to Her Newborn. Jama 2020.

17. Chen H, Guo J, Wang C, Luo F, Yu X, Zhang W, Li J, Zhao D, Xu D, Gong Q et al: Clinical characteristics and intrauterine vertical transmission potential of COVID-19 infection in nine pregnant women: a retrospective review of medical records. Lancet (London, England) 2020, 395(10226):809815.

238 18. Sepkowitz KA: Nosocomial infections in patients with cancer. Lancet Oncology 2009, 10(6):589-597.

240 19. Liang W, Guan W, Chen R, Wang W, Li J, Xu K, Li C, Ai Q, Lu W, Liang H et $a l$ : Cancer patients in SARS-CoV-2 infection: a nationwide analysis in China. The Lancet Oncology 2020, 21(3):335-337.

243 20. Jie-Yao, Li, Xiu-Fang, Duan, Li-Ping, Wang, Yu-Jie, Xu, Lan, Huang: Selective depletion of regulatory $T$ cell subsets by docetaxel treatment in patients with nonsmall cell lung cancer. Journal of Immunology Research 2014.

246 21. Weinstein JN, Collisson EA, Mills GB, Shaw KR, Ozenberger BA, Ellrott K, 

analysis project. Nature genetics 2013, 45(10):1113-1120.

22. Dunham I, Kundaje A, Aldred SF, Collins PJ, Davis CA, Doyle F, Epstein CB, Frietze S, Harrow J, Kaul R et al: An integrated encyclopedia of DNA elements in the human genome. Nature 2012, 489(7414):57-74.

23. Wang Q, Zhang Y, Wu L, Niu S, Song C, Zhang Z, Lu G, Qiao C, Hu Y, Yuen KY et al: Structural and Functional Basis of SARS-CoV-2 Entry by Using Human ACE2. Cell 2020.

24. Hoffmann M, Kleine-Weber H, Schroeder S, Krüger N, Herrler T, Erichsen S, Schiergens TS, Herrler G, Wu N-H, Nitsche A et al: SARS-CoV-2 Cell Entry Depends on ACE2 and TMPRSS2 and Is Blocked by a Clinically Proven Protease Inhibitor. Cell 2020.

25. Holmes KV: Human Coronavirus HCoV-229E Enters Susceptible Cells via the Endocytic Pathway. Oxygen Transport to Tissue XXXIII 2001, 494:193-

26. Inoue $\mathrm{Y}$, Tanaka N, Tanaka $\mathrm{Y}$, Inoue S, Morita $\mathrm{K}$, Zhuang M, Hattori T, entry into host cells through a novel clathrin- and caveolae-independent 
endocytic pathway. Cell research 2008, 18(2):290-301.

269 28. Jia HP, Look DC, Tan P, Shi L, Hickey M, Gakhar L, Chappell MC, WohlfordLenane C, McCray PB, Jr.: Ectodomain shedding of angiotensin converting enzyme 2 in human airway epithelia. American journal of physiology Lung cellular and molecular physiology 2009, 297(1):L84-96.

273 29. Lambert DW, Yarski M, Warner FJ, Thornhill P, Parkin ET, Smith AI, Hooper NM, Turner AJ: Tumor necrosis factor-alpha convertase (ADAM17) mediates regulated ectodomain shedding of the severe-acute respiratory syndrome-coronavirus (SARS-CoV) receptor, angiotensin-converting enzyme-2 (ACE2). The Journal of biological chemistry 2005, 280(34):3011330119. 
Figures

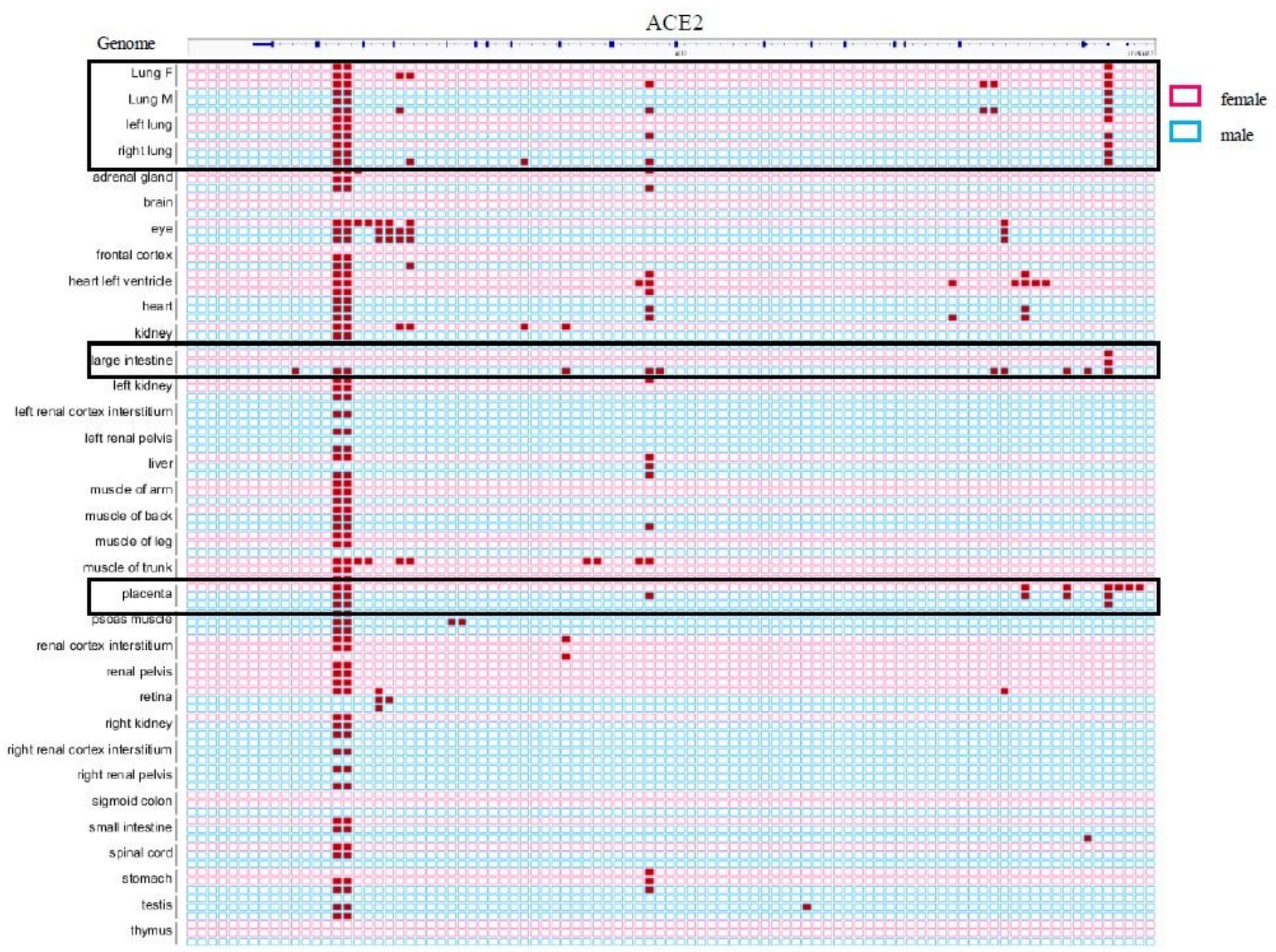

chrX: $15576000-15622500$

\section{Figure 1}

Landscape of open chromatin for the ACE2 gene in tissues Genomic region chrX:15576000622500(human genome: hg19), covering the ACE2 gene locus, was split by 500bp/bin. ACE2 accessible regions were identified as genomic bins overlapping with DH Speaks for each tissue. Accessible regions at the ACE2 promoter site were specifically open in tissue from the lung, large intestine ,and placenta (black boxes). 
A

Tumor Tissues

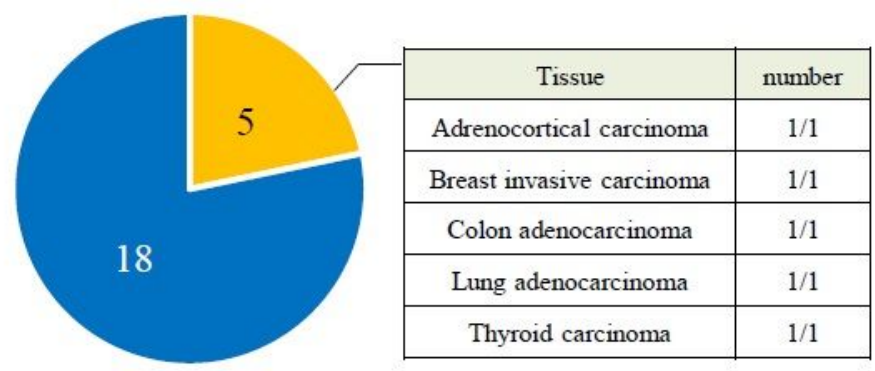

Normal cell lines

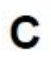

\begin{tabular}{|c|c|c|}
\hline \multirow{7}{*}{115} & Cell line & number \\
\hline & primary tracheal epithelial cells & $1 / 1$ \\
\hline & small airway epithelial cells & $2 / 2$ \\
\hline & bronchial epithelial cells & $2 / 2$ \\
\hline & B cells & $1 / 5$ \\
\hline & lymphoblastoid cell & $1 / 32$ \\
\hline & iPS cells & $1 / 12$ \\
\hline \multirow{3}{*}{ DHS detected } & embryonic stem cells & $1 / 19$ \\
\hline & esophageal epithelial cells & $3 / 3$ \\
\hline & mammary epithelial cells & $2 / 5$ \\
\hline \multirow{2}{*}{ No DHS signal } & pancreatic duct cells & $1 / 3$ \\
\hline & prostate epithelial cell & $1 / 8$ \\
\hline
\end{tabular}

B

Cancer cell lines

\begin{tabular}{|c|c|}
\hline Cell line & number \\
\hline ductal carcinoma epithelial & $5 / 8$ \\
\hline mammary gland adenocarcinoma & $9 / 22$ \\
\hline lung adenocarcinoma & $2 / 2$ \\
\hline cervical carcinoma & $2 / 11$ \\
\hline colorectal adenocarcinoma & $1 / 5$ \\
\hline endometrial adenocarcinoma & $3 / 7$ \\
\hline endometrium adenocarcinoma & $2 / 4$ \\
\hline epithelial & $1 / 10$ \\
\hline Non-small-cell carcinoma & $6 / 8$ \\
\hline hepatocellular carcinoma & $1 / 2$ \\
\hline
\end{tabular}

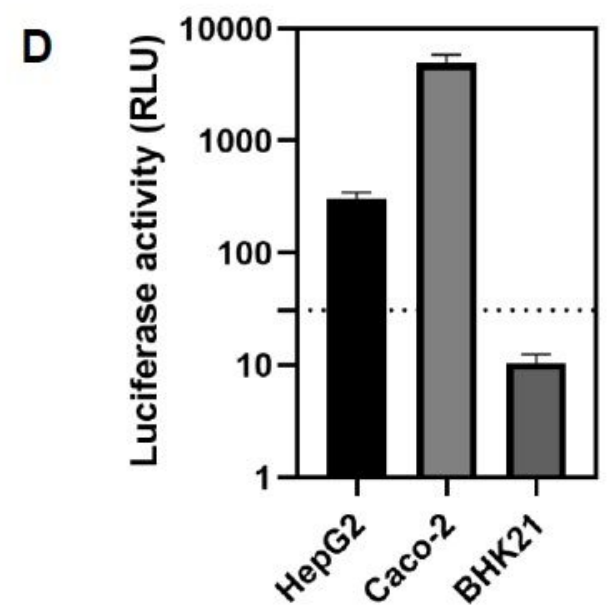

Figure 2

Open chromatin for the ACE2 promoter in different tissues and cell lines. (A) DNase-seq peaks detected in the ACE2 promoter for tumor tissues obtained from TCGA project. (B, C) DNase-seq peaks detected in the ACE2 promoter for cancer cell lines (B) and normal cell lines (C) obtained from the ENCODE project. (D) The HepG2 and Caco-2 cell lines with DNase-seq peaks in the ACE2 promoter were susceptible to SARSCoV-2 pseudovirus. Susceptibility was defined by luciferase activity exceeding three times that in unsusceptible BHK21 cells (dashed line). 


\section{Accessible promoter mediated compensatory effect of $A C E 2$ provides new insights into the tissue tropism of SARS-CoV-2}

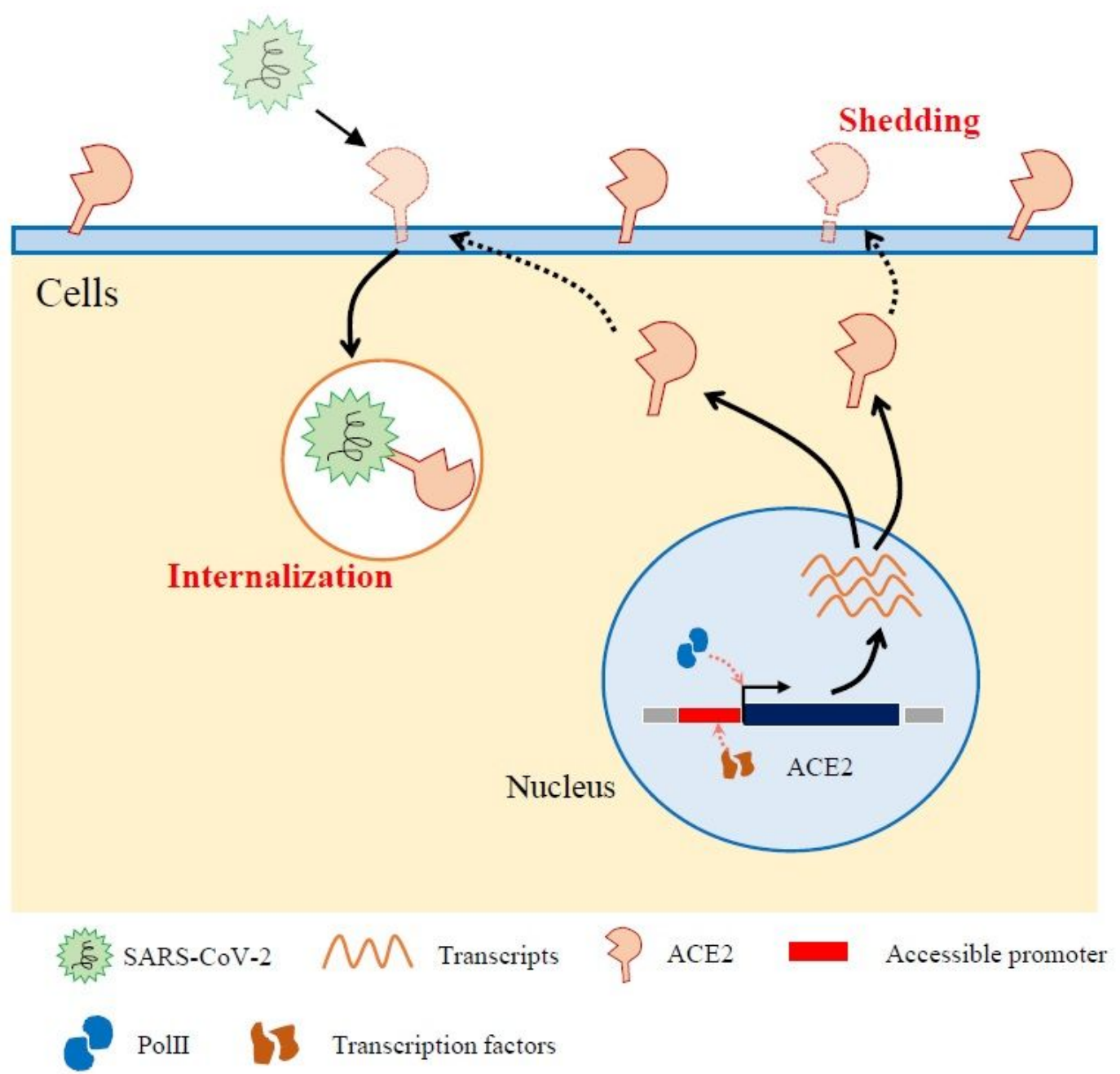

Figure 3

Hypothetical model in which open chromatin at the promoter mediates ACE2 supplementation and ensures the entry of SARS-CoV-2

\section{Supplementary Files}

This is a list of supplementary files associated with this preprint. Click to download.

- SupplementalFigure.pdf

- TableS1.xIsx

- Tables2.xlsx

- TableS3.xIsx 
- Tables4.xIsx

- TableS5.xIsx 